\title{
Thomas Ferretti
}

Doctorant en philosophie, Université Catholique de Louvain, Belgique

(2012)

LE DEVOIR DE PHILO

\section{"John Stuart Mill \\ et l’Année internationale des coopératives.}

D'après le philosophe, ces institutions sont un moyen de garantir la dignité de tous et de promouvoir les valeurs démocratiques tout en conservant une économie efficace.”

\author{
Un document produit en version numérique par Jean-Marie Tremblay, bénévole, \\ professeur de sociologie retraité du Cégep de Chicoutimi \\ Courriel: jean-marie_tremblay@uqac.ca \\ Site web pédagogique : http://www.uqac.ca/jmt-sociologue/ \\ Dans le cadre de: "Les classiques des sciences sociales" \\ Une bibliothèque numérique fondée et dirigée par Jean-Marie Tremblay, \\ professeur de sociologie au Cégep de Chicoutimi \\ Site web: http://classiques.uqac.ca/ \\ Une collection développée en collaboration avec la Bibliothèque \\ Paul-Émile-Boulet de l'Université du Québec à Chicoutimi \\ Site web: http://bibliotheque.uqac.ca/
}




\section{Politique d'utilisation de la bibliothèque des Classiques}

Toute reproduction et rediffusion de nos fichiers est interdite, même avec la mention de leur provenance, sans l'autorisation formelle, écrite, du fondateur des Classiques des sciences sociales, Jean-Marie Tremblay, sociologue.

Les fichiers des Classiques des sciences sociales ne peuvent sans autorisation formelle:

- être hébergés (en fichier ou page web, en totalité ou en partie) sur un serveur autre que celui des Classiques.

- servir de base de travail à un autre fichier modifié ensuite par tout autre moyen (couleur, police, mise en page, extraits, support, etc...),

Les fichiers (.html, .doc, .pdf, .rtf, .jpg, .gif) disponibles sur le site Les Classiques des sciences sociales sont la propriété des Classiques des sciences sociales, un organisme à but non lucratif composé exclusivement de bénévoles.

Ils sont disponibles pour une utilisation intellectuelle et personnelle et, en aucun cas, commerciale. Toute utilisation à des fins commerciales des fichiers sur ce site est strictement interdite et toute rediffusion est également strictement interdite.

L'accès à notre travail est libre et gratuit à tous les utilisateurs. C'est notre mission.

Jean-Marie Tremblay, sociologue

Fondateur et Président-directeur général, LES CLASSIQUES DES SCIENCES SOCIALES. 
Cette édition électronique a été réalisée par Jean-Marie Tremblay, bénévole, professeur de sociologie au Cégep de Chicoutimi à partir de :

\section{Thomas FERRETTI}

[doctorant en philosophie, Université catholique de Louvain, Belgique.]

\section{“John Stuart Mill et l’Année internationale des coopératives.”}

D’après le philosophe, ces institutions sont un moyen de garantir la dignité de tous et de promouvoir les valeurs démocratiques tout en conservant une économie efficace.”

Un article publié dans $\mathbf{L e} \boldsymbol{D e}$ voir, Montréal, édition du samedi, LE DEVOIR.com 20 octobre 2012, page B6 — Le devoir de philo.

[Autorisation formelle accordée par l'auteur le 21 octobre 2012 de diffuser ce texte dans Les Classiques des sciences sociales.]

\section{Courriel : thomas.ferretti1@gmail.com}

Polices de caractères utilisée : Times New Roman, 14 points.

Édition électronique réalisée avec le traitement de textes Microsoft Word 2008 pour Macintosh.

Mise en page sur papier format : LETTRE US, 8.5’’ x 11’.

Édition numérique réalisée le 22 octobre 2012 à Chicoutimi, Ville de Saguenay, Québec. 


\section{Thomas FERRETTI}

[doctorant en philosophie, Université catholique de Louvain, Belgique.]

\section{“John Stuart Mill et l’Année internationale des coopératives.”}

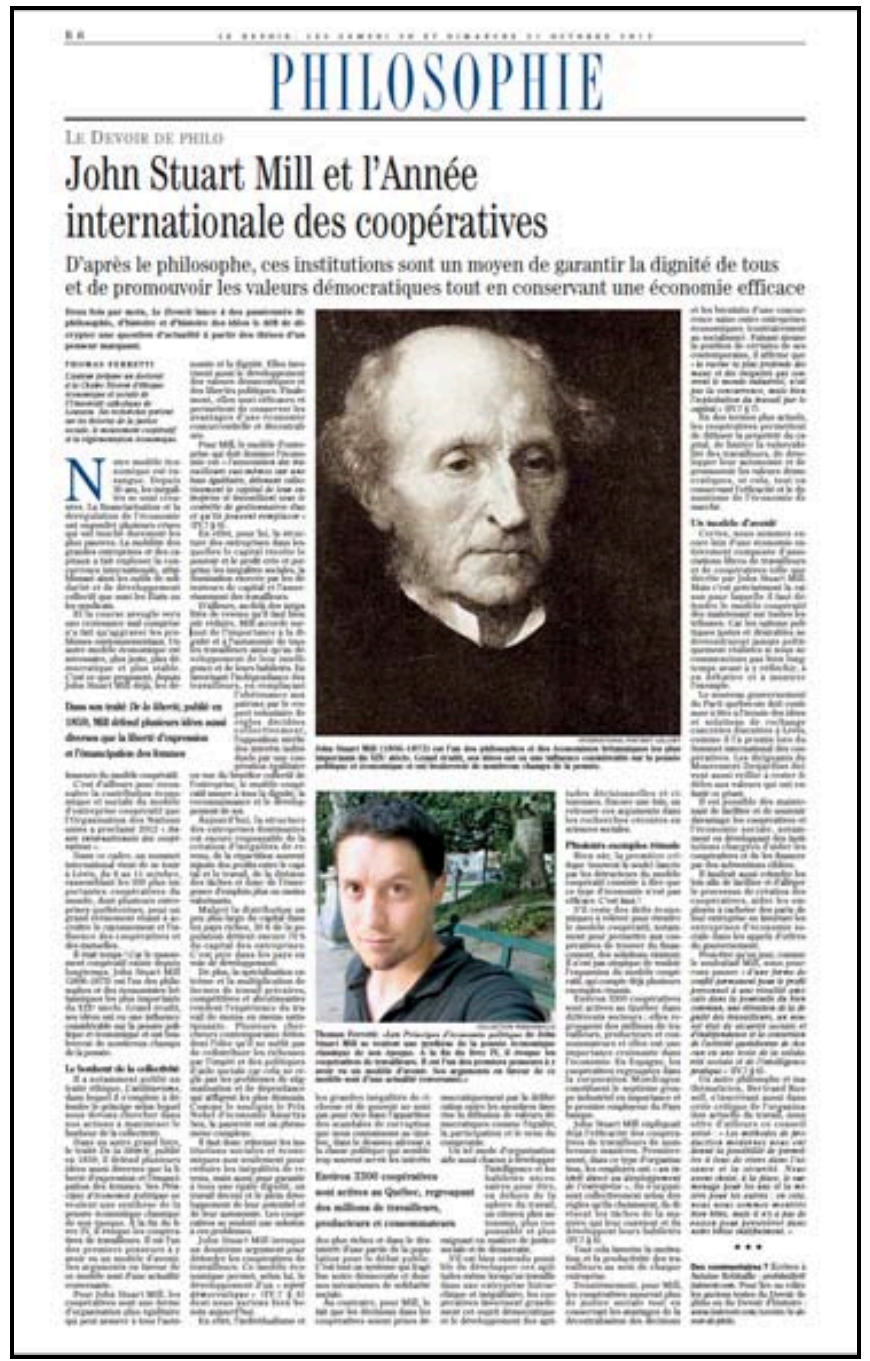

Un article publié dans Le Devoir, Montréal, édition du samedi, 20 octobre 2012, page B6 — Le devoir de philo. 
Thomas FERRETTI

[doctorant en philosophie, Université catholique de Louvain, Belgique.]

\section{“John Stuart Mill \\ et l’Année internationale des coopératives.}

D’après le philosophe, ces institutions sont un moyen de garantir la dignité de tous et de promouvoir les valeurs démocratiques tout en conservant une économie efficace.”

Un article publié dans Le Devoir, Montréal, édition du samedi, 20 octobre 2012, page B6 — Le devoir de philo.

\section{À RETENIR}

Deux fois par mois, Le Devoir lance à des passionnés de philosophie, d'histoire et d'histoire des idées le défi de décrypter une question d'actualité à partir des thèses d'un penseur marquant.

Notre modèle économique est exsangue. Depuis 30 ans, les inégalités se sont creusées. La financiarisation et la dérégulation de l'économie ont engendré plusieurs crises qui ont touché durement les plus pauvres. La mobilité des grandes entreprises et des capitaux a fait exploser la concurrence internationale, affaiblissant ainsi les outils de solidarité et de développement collectif que sont les États ou les syndicats.

Et la course aveugle vers une croissance mal comprise n'a fait qu'aggraver les problèmes environnementaux. Un autre modèle économique est nécessaire, plus juste, plus démocratique et plus stable. C’est ce que proposent, depuis John Stuart Mill déjà, les défenseurs du modèle coopératif. 


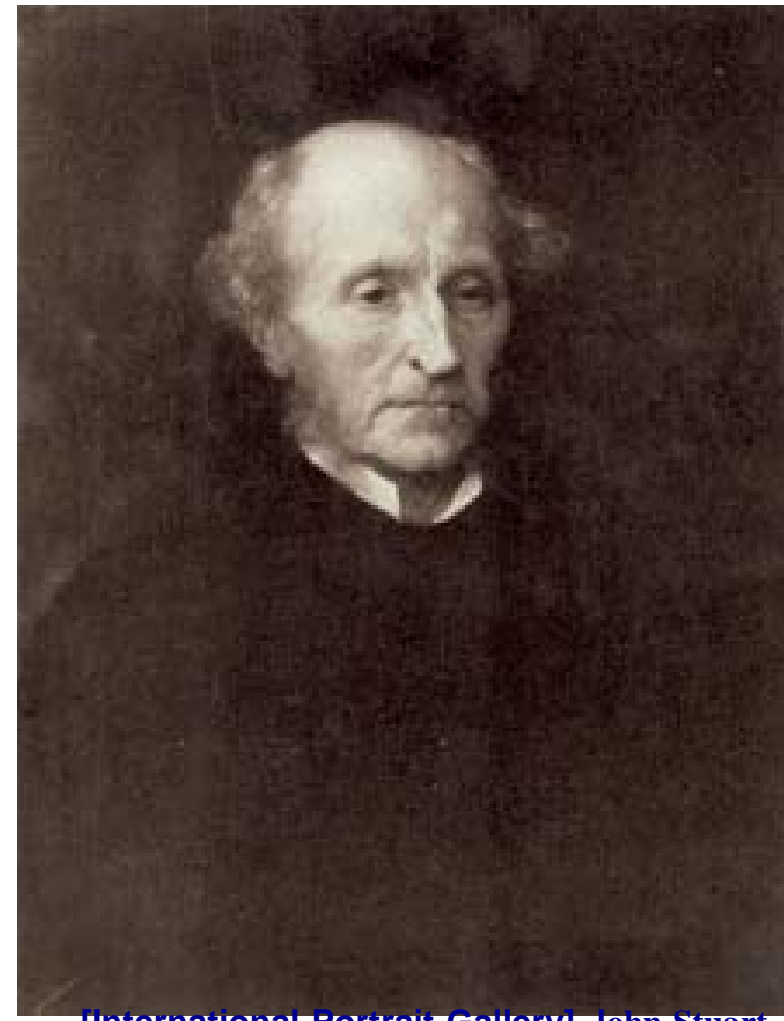

[International Portrait Gallery] John Stuart Mill (1806-1873) est l'un des philosophes et des économistes britanniques les plus importants du XIXe siècle. Grand érudit, ses idées ont eu une influence considérable sur la pensée politique et économique et ont bouleversé de nombreux champs de la pensée.
C'est d'ailleurs pour reconnaître la contribution économique et sociale du modèle d'entreprise coopératif que l'Organisation des Nations unies a proclamé 2012 «Année internationale des coopératives ».

Dans son traité De la liberté, publié en 1859, Mill défend plusieurs idées aussi diverses que la liberté d'expression et l'émancipation des femmes.

Dans ce cadre, un sommet international vient de se tenir à Lévis, du 8 au 11 octobre, rassemblant les 300 plus importantes coopératives du monde, dont plusieurs entreprises québécoises, pour un grand événement visant à accroître le rayonnement et l'influence des coopératives et des mutuelles.

Il était temps ! Car le mouvement coopératif existe depuis longtemps. John Stuart Mill (1806 -1873) est l'un des philosophes et des économistes britanniques les plus importants du XIXe siècle. Grand érudit, ses idées ont eu une influence considérable sur la pensée politique et économique et ont bouleversé de nombreux champs de la pensée. 


\section{Le bonheur de la collectivité}

Il a notamment publié un traité éthique, L’utilitarisme, dans lequel il s'emploie à défendre le principe selon lequel nous devons chercher dans nos actions à maximiser le bonheur de la collectivité.

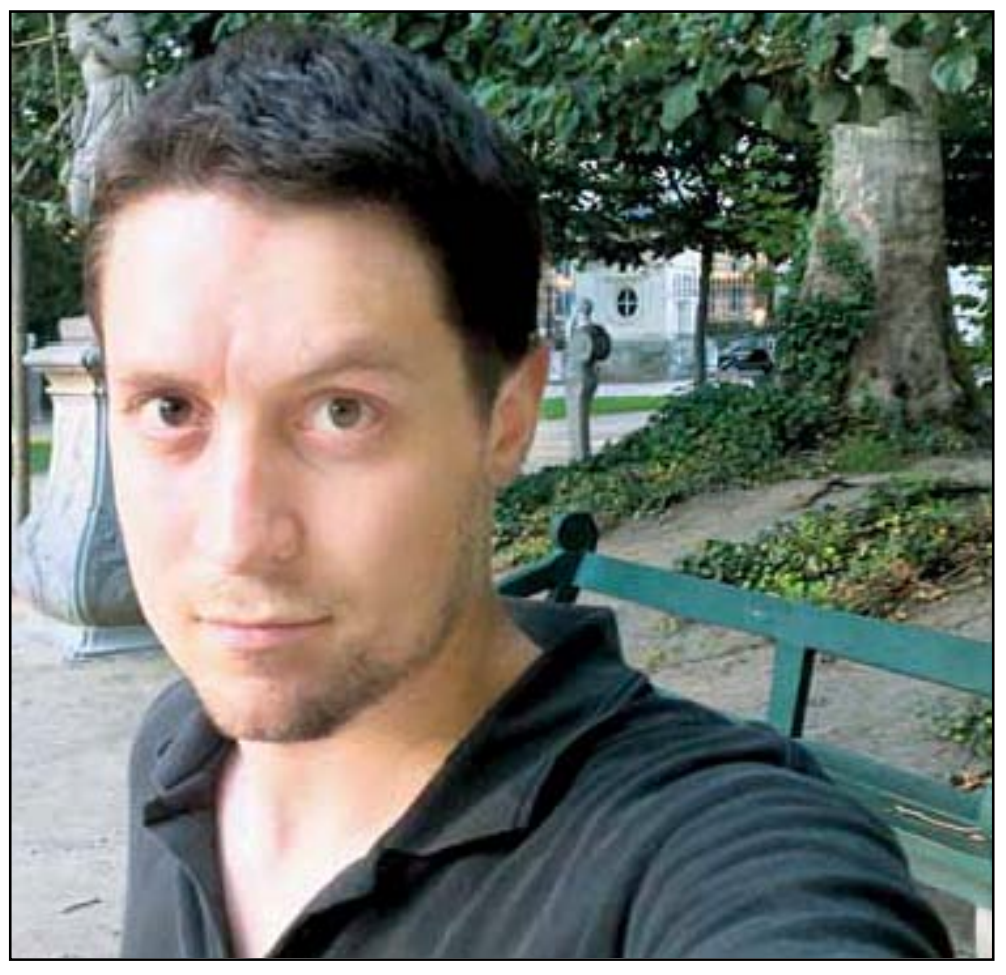

[Collection personnelle] Thomas Ferretti : «Les Principes d'économie politique de John Stuart Mill se veulent une synthèse de la pensée économique classique de son époque. À la fin du livre IV, il évoque les coopératives de travailleurs. II est l'un des premiers penseurs à y avoir vu un modèle d'avenir. Ses arguments en faveur de ce modèle sont d'une actualité ren-

Dans un autre grand livre, le traité De la liberté, publié en 1859, il défend plusieurs idées aussi diverses que la liberté d'expression et l'émancipation des femmes. Ses Principes d'économie politique se veulent une synthèse de la pensée économique classique de son époque. À la fin du livre IV, il évoque les coopératives de travailleurs. Il est l'un des premiers penseurs à y avoir vu un modèle d'avenir. Ses arguments en faveur de ce modèle sont d'une actualité renversante.

Pour John Stuart Mill, les coopératives sont une forme d'organisation plus égalitaire qui peut assurer à tous l'autonomie et la dignité. Elles favorisent aussi le développement des valeurs démocratiques et des libertés politiques. Finalement, elles sont efficaces et permettent de conserver les avantages d'une économie concurrentielle et décentralisée.

Pour Mill, le modèle d'entreprise qui doit dominer l'économie est « l'association des travailleurs eux-mêmes sur une base égalitaire, détenant collectivement le capital de leur entreprise et travaillant sous le contrôle de gestionnaires élus et qu'ils peuvent remplacer » (IV.7 § 6). 
En effet, pour lui, la structure des entreprises dans lesquelles le capital récolte le pouvoir et le profit crée et perpétue les inégalités sociales, la domination exercée par les détenteurs de capital et l'asservissement des travailleurs.

D’ailleurs, au-delà des inégalités de revenu qu'il faut bien sûr réduire, Mill accorde surtout de l'importance à la dignité et à l'autonomie de tous les travailleurs ainsi qu'au développement de leur intelligence et de leurs habiletés. En favorisant l'indépendance des travailleurs, en remplaçant l'obéissance aux patrons par le respect volontaire de règles décidées collectivement, l’opposition stérile des intérêts individuels par une coopération égalitaire en vue du bénéfice collectif de l'entreprise, le modèle coopératif assure à tous la dignité, la reconnaissance et le développement de soi.

Aujourd'hui, la structure des entreprises dominantes est encore responsable de la création d'inégalités de revenu, de la répartition souvent injuste des profits entre le capital et le travail, de la division des tâches et donc de l'émergence d'emplois plus ou moins valorisants.

Malgré la distribution un peu plus large du capital dans les pays riches, 30\% de la population détient encore $70 \%$ du capital des entreprises. C’est pire dans les pays en voie de développement.

De plus, la spécialisation extrême et la multiplication de formes de travail précaires, compétitives et abrutissantes rendent l'expérience du travail de moins en moins satisfaisante. Plusieurs chercheurs contemporains défendent l'idée qu'il ne suffit pas de redistribuer les richesses par l'impôt et des politiques d'aide sociale car cela ne règle pas les problèmes de stigmatisation et de dépendance qui affligent les plus démunis. Comme le souligne le Prix Nobel d'économie Amartya Sen, la pauvreté est un phénomène complexe.

Il faut donc réformer les institutions sociales et économiques non seulement pour réduire les inégalités de revenu, mais aussi pour garantir à tous une égale dignité, un travail décent et le plein développement de leur potentiel et de leur autonomie. Les coopératives se veulent une solution à ces problèmes.

John Stuart Mill invoque un deuxième argument pour défendre les coopératives de travailleurs. Ce modèle économique permet, selon lui, 
le développement d’un «esprit démocratique » (IV.7 § 6) dont nous aurions bien besoin aujourd'hui.

En effet, l'individualisme et les grandes inégalités de richesse et de pouvoir ne sont pas pour rien dans l'apparition des scandales de corruption que nous connaissons au Québec, dans le désaveu adressé à la classe politique qui semble trop souvent servir les intérêts des plus riches et dans le désintérêt d'une partie de la population pour le débat public. C'est tout un système qui fragilise notre démocratie et donc nos mécanismes de solidarité sociale.

\section{Environ 3300 coopératives sont actives au Québec, regroupant des millions de travail- leurs, producteurs et consommateurs.}

Au contraire, pour Mill, le fait que les décisions dans les coopératives soient prises démocratiquement par la délibération entre les membres favorise la diffusion de valeurs démocratiques comme l'égalité, la participation et le sens du compromis.

Un tel mode d'organisation aide aussi chacun à développer l'intelligence et les habiletés nécessaires pour être, en dehors de la sphère du travail, un citoyen plus autonome, plus responsable et plus exigeant en matière de justice sociale et de démocratie.

S’il est bien entendu possible de développer ces aptitudes même lorsqu'on travaille dans une entreprise hiérarchique et inégalitaire, les coopératives favorisent grandement cet esprit démocratique et le développement des aptitudes décisionnelles et citoyennes. Encore une fois, on retrouve ces arguments dans les recherches récentes en sciences sociales. 


\section{Plusieurs exemples réussis}

Bien sûr, la première critique (souvent la seule) lancée par les détracteurs du modèle coopératif consiste à dire que ce type d'économie n'est pas efficace. C'est faux !

S’il reste des défis économiques à relever pour étendre le modèle coopératif, notamment pour permettre aux coopératives de trouver du financement, des solutions existent. Il n'est pas utopique de vouloir l'expansion du modèle coopératif, qui compte déjà plusieurs exemples réussis.

Environ 3300 coopératives sont actives au Québec dans différents secteurs ; elles regroupent des millions de travailleurs, producteurs et consommateurs et elles ont une importance croissante dans l'économie. En Espagne, les coopératives regroupées dans la corporation Mondragon constituent le septième groupe industriel en importance et le premier employeur du Pays basque.

John Stuart Mill expliquait déjà l'efficacité des coopératives de travailleurs de nombreuses manières. Premièrement, dans ce type d'organisation, les employés ont « un intérêt direct au développement de l'entreprise ", ils s'organisent collectivement selon des règles qu'ils choisissent, ils divisent les tâches de la manière qui leur convient et ils développent leurs habiletés (IV.7 § 6).

Tout cela favorise la motivation et la productivité des travailleurs au sein de chaque entreprise.

Deuxièmement, pour Mill, les coopératives assurent plus de justice sociale tout en conservant les avantages de la décentralisation des décisions et les bienfaits d'une concurrence saine entre entreprises économiques (contrairement au socialisme). Faisant sienne la position de certains de ses contemporains, il affirme que « la racine la plus profonde des maux et des iniquités qui couvrent le monde industriel, n'est pas la concurrence, mais bien l'exploitation du travail par le capital » (IV.7 § 7).

En des termes plus actuels, les coopératives permettent de diffuser la propriété du capital, de limiter la vulnérabilité des travailleurs, de 
développer leur autonomie et de promouvoir les valeurs démocratiques, et cela, tout en conservant l'efficacité et le dynamisme de l’économie de marché.

\section{Un modèle d'avenir}

Certes, nous sommes encore loin d'une économie entièrement composée d'associations libres de travailleurs et de coopératives telle que décrite par John Stuart Mill. Mais c’est précisément la raison pour laquelle il faut défendre le modèle coopératif dès maintenant sur toutes les tribunes. Car les options politiques justes et désirables ne deviendraient jamais politiquement réalistes si nous ne commencions pas bien longtemps avant à y réfléchir, à en débattre et à montrer l'exemple.

Le nouveau gouvernement du Parti québécois doit continuer à être à l'écoute des idées et solutions de rechange concrètes discutées à Lévis, comme il l'a promis lors du Sommet international des coopératives. Les dirigeants du Mouvement Desjardins doivent aussi veiller à rester fidèles aux valeurs qui ont enfanté ce géant.

Il est possible dès maintenant de faciliter et de soutenir davantage les coopératives et l'économie sociale, notamment en développant des institutions chargées d'aider les coopératives et de les financer par des subventions ciblées.

Il faudrait aussi refondre les lois afin de faciliter et d'alléger le processus de création des coopératives, aider les employés à racheter des parts de leur entreprise ou favoriser les entreprises d'économie sociale dans les appels d'offres du gouvernement.

Peut-être qu'un jour, comme le souhaitait Mill, nous pourrons passer « d'une forme de conflit permanent pour le profit personnel à une rivalité amicale dans la poursuite du bien commun, une élévation de la dignité des travailleurs, un nouvel état de sécurité sociale et d'indépendance et la conversion de l'activité quotidienne de chacun en une école de la solidarité sociale et de l'intelligence pratique » (IV.7 § 6). 
Un autre philosophe et mathématicien, Bertrand Russell, s'inscrivant aussi dans cette critique de l'organisation actuelle du travail, nous offre d'ailleurs ce conseil avisé : « Les méthodes de production modernes nous ont donné la possibilité de permettre à tous de vivre dans l'aisance et la sécurité. Nous avons choisi, à la place, le surmenage pour les uns et la misère pour les autres: en cela, nous nous sommes montrés bien bêtes, mais il n’y a pas de raison pour persévérer dans notre bêtise indéfiniment. "

L'auteur prépare un doctorat à la Chaire Hoover d'éthique économique et sociale de l'Université catholique de Louvain. Ses recherches portent sur les théories de la justice sociale, le mouvement coopératif et la réglementation économique.

Des commentaires ? Écrivez à Antoine Robitaille

[arobitaille@ledevoir.com].

Pour lire ou relire les anciens textes du Devoir de philo ou du Devoir d'histoire :

http://www.ledevoir.com/societe/le-devoir-de-philo.

\section{Fin du texte}

